\title{
Morbimortalidad cardiovascular y variación del grado funcional en pacientes ancianos portadores de marcapasos
}

\author{
Medium-long term mortality and change in functional status in elderly patients \\ with pacemaker
}

\author{
Pedro Pérez-Díaz ${ }^{1 *}$, Javier Jiménez-Díaz ${ }^{1}$, Felipe Higuera-Sobrino ${ }^{1}$, Jesús Piqueras-Flores ${ }^{1}$, \\ Raquel Frías-García1 ${ }^{1}$ Virginia Mazoteras-Muñoz², Ramón Maseda-Uriza ${ }^{1}$ y Violeta Arenas-Cambronero ${ }^{3}$ \\ ${ }^{1}$ Servicio de Cardiología; ${ }^{2}$ Servicio de Geriatría; ${ }^{3}$ Unidad Docente de Medicina de Atención Familiar y Comunitaria (UDMAFYC); Hospital General \\ Universitario de Ciudad Real (HGUCR). Ciudad Real, España.
}

\begin{abstract}
Resumen
Antecedentes: Aproximadamente un 49\% de los implantes se efectúan a individuos mayores de 80 años; sin embargo, la evidencia científica sobre mortalidad y cambio en la situación funcional de estos pacientes es muy pobre. Objetivo: Diseñamos un estudio prospectivo para analizar la morbimortalidad cardiovascular y la variación de su grado funcional a medio plazo en pacientes ancianos con electroestimulación permanente. Método: Estudio observacional prospectivo, que incluye 308 pacientes ancianos sometidos a implante de marcapasos en un hospital terciario entre 2012 y 2014. Como variables principales se evaIuaron eventos cardiovasculares, mortalidad y grado funcional, con una media de seguimiento de 3.5 años. Resultados: El 60\% de los pacientes incluidos en nuestro estudio tenían una edad superior a 80 años, y la indicación más frecuente fue el bloqueo auriculoventricular completo (44.3\%), seguido de la fibrilación auricular lenta o bloqueada (16.7\%). El modo de estimulación más frecuente en la muestra general fue el DDD (38.6\%) (VVI en pacientes octogenarios, 38.7\%). En el seguimiento, la mortalidad a largo plazo fue mayor en dispositivos ventriculares, especialmente en octogenarios $(p=0.001)$. El modo de estimulación ventricular (VVI) fue predictor independiente de mortalidad. A largo plazo, no se observó mejoría del índice de Barthel ni del grado funcional tras el implante del marcapasos. Conclusiones: La morbimortalidad cardiovascular en pacientes octogenarios portadores de marcapasos resulta superior a la de la población general, especialmente en dispositivos monocamerales. La electroestimulación permanente no se asocia con mejoría del grado funcional a medio-largo plazo en estos pacientes.
\end{abstract}

Palabras clave: Marcapasos. Octogenarios. Bloqueo aurculoventricular completo. Grado funcional. Mortalidad. España.

\begin{abstract}
Background: Nowadays, $49 \%$ of patients with pacemakers are older than 80 years old. Nevertheless, mortality and change in functional status after pacemaker implantation are not well documented in elderly patients. Objective: We designed a prospective study to analyze cardiovascular mortality and change in functional status of elderly patients, medium-long term
\end{abstract}

\footnotetext{
Correspondencia:

*Pedro Pérez-Díaz

Hospital General Universitario de Ciudad Real (HGUCR)

Servicio de cardiología (5. ${ }^{a}$ B)

Calle del Obispo Rafael Torija, s/n

Fecha de recepción: 30-09-2018

Fecha de aceptación: 14-01-2019

Disponible en internet: 02-10-2019

13005 Ciudad Real, España

DOI: $10.24875 /$ ACM.M19000053

Arch Cardiol Mex. 2019;89(3):233-241

www.archivoscardiologia.com

1405-9940 @ 2019 Instituto Nacional de Cardiología Ignacio Chávez. Publicado por Permanyer México SA de CV. Este es un artículo Open Access bajo la licencia CC BY-NC-ND (http://creativecommons.org/licenses/by-nc-nd/4.0/).
} 
after pacemaker implantation. Methods: Observational study including pacemaker implants in individual older than 70 years old in a single center university hospital between 2012 and 2014. Analysis testing for an association between pacemaker system, medium-long term mortality and functional status after implantation were undertaken. Results: $60 \%$ of patients were older than 80 years old. Third-degree atrio-ventricular block (44.3\%) and slow ventricular response atrial fibrillation (16.7\%) were the most frequent electrocardiogram abnormalities, while bicameral DDD was the sort of pacing our department used the most (38.6\%) (VVI in octogenarian patients, 38.7\%). Long-term mortality was significantly higher in ventricular devices, especially in octogenarian patients ( $p=0.001$ respectively). Single-chamber VVI pacing acted as independent predictors of all-cause mortality in these individuals $(p=0.001)$. We found no significant improvement in Barthel index and functional status in this subgroup of patients, 3 years after pacing. Conclusion: Long-term mortality in individuals older than 80 years old with pacemaker implantation, was significantly higher comparing with general population, especially in ventricular devices. No significant improvement in functional status was detected in this subgroup of patients.

Key words: Pacemaker. Octogenarian. Third-degree atrio-ventricular block. Functional status. Mortality. Spain.

\section{Introducción}

Los marcapasos permanentes son el tratamiento de elección de las bradiarritmias significativas, producidas generalmente por la degeneración del sistema de excitación-conducción cardíaco asociado a la edad avanzada. Las guías de electroestimulación de la Sociedad Europea de Cardiología de 2013 establecieron la disfunción sinusal sintomática, bloqueo auriculoventricular (BAV) de segundo grado Móbitz I sintomático, grado Móbitz II y de tercer grado, como indicaciones fundamentales de marcapasos permanente ${ }^{1}$. Las indicaciones más frecuentes de implante de marcapasos permanente son el BAV de tercer grado, seguido de la disfunción sinusal sintomática, tendencia que se ha mantenido estable a lo largo de los últimos años. Sin embargo, por el incremento progresivo de la esperanza de vida que ha tenido lugar en España en los últimos años, la edad media en el momento del implante de marcapasos ha aumentado de 77 años en 2012 hasta 77.8 años en $2016^{2}$. El $50 \%$ del total de primoimplantes de marcapasos corresponde a individuos mayores de 80 años. A pesar de esto, la mayoría de la evidencia científica disponible se basa en estudios realizados con pacientes de edad media inferior a la edad de la población atendida habitualmente en las unidades de arritmias, y algunos de ellos incluso excluyen este subgrupo de pacientes ${ }^{3-7}$.

Si bien el modo de estimulación ventricular (VVI) está indicado en pacientes con fibrilación auricular y BAV, también se emplea en pacientes con BAV avanzado con elevada comorbilidad o esperanza de vida limita$\mathrm{da}^{1}$. Los dispositivos fisiológicos, como el AAI, el VDD y el DDD, que permiten una estimulación secuencial que mantiene la sincrónica auriculoventricular, se implantan en individuos con disfunción sinusal sintomática o BAV avanzado. En España, el modo de estimulación más ampliamente utilizado es el DDD (47.2\%), seguido del VVI (39.3\%) y, a una amplia distancia, el VDD $(10.2 \%)^{2}$.

Actualmente existe controversia acerca de los beneficios de la estimulación fisiológica en comparación con la estimulación ventricular en términos de morbimortalidad. La estimulación ventricular se ha asociado con mayor incidencia de fibrilación auricular e insuficiencia cardíaca que la estimulación fisiológica tal como pusieron de manifiesto los estudios DANPACE y MOST ${ }^{8,9}$. Sin embargo, ningún estudio ha mostrado diferencias concluyentes en cuanto a la mortalidad 3 ,10-22.

Por otro lado, los resultados en mejoría de calidad de vida y grado funcional en pacientes ancianos tras el implante de marcapasos son contradictorios. El estudio PASE demostró mejoría significativa en la calidad de vida a los tres meses post-implante de marcapasos, independientemente de la estimulación uni- o bicame$\mathrm{ral}^{23}$. Sin embargo, estos hallazgos no se han confirmado en otros estudios ${ }^{24-26}$.

El objetivo principal de nuestro estudio consistió en analizar la mortalidad cardiovascular y global a medio plazo de pacientes ancianos con electroestimulación permanente. Los objetivos secundarios que buscamos fue la descripción de los eventos cardiovasculares y la variación de su grado funcional antes y después del implante del marcapasos.

\section{Materiales y métodos}

Estudio observacional prospectivo que incluyó 308 pacientes consecutivos mayores de 70 años y sometidos a un implante electivo de marcapasos permanente entre enero de 2012 y diciembre de 2014 en la Unidad de Arritmias del Hospital General Universitario de Ciudad Real. Los criterios de inclusión fueron: pacientes mayores de 70 años con indicación de marcapasos 
permanente según las guías actuales de estimulación cardíaca de la Sociedad Europea de Cardiología ${ }^{1}$. Se excluyeron los pacientes menores de 70 años, pacientes sometidos a implante de resincronizador o desfibrilador, pacientes con fracción de eyección del ventrículo izquierdo (FEVI) menor del $35 \%$ y pacientes sometidos a recambio de generador.

\section{Características basales y seguimiento}

Analizamos las características basales incluyendo diabetes mellitus, dislipemia, hipertensión arterial, enfermedad pulmonar obstructiva crónica, fibrilación auricular, FEVI, filtrado glomerular (según fórmula Chronic Kidney Disease Epidemiology Collaboration [CKD-EPI]), indicaciones del primoimplante y el tipo de electroestimulación (ventricular, en el caso de VVI, o fisiológica, en el caso de AAI, VDD o DDD). Las complicaciones periprocedimiento que registramos fueron: dislocación del electrodo auricular/ventricular, perforación cardíaca, neumotórax, hematoma que precisó transfusión, e infección del sistema de marcapasos. Además, se realizó la valoración funcional geriátrica al ingreso y a los 3 años del implante del dispositivo mediante el Índice funcional de Barthel sobre puntuación de 100 y la Escala funcional de la Cruz Roja (CRF) ${ }^{27,28}$. La variable principal fue la mortalidad por cualquier causa. Las variables secundarias fueron complicaciones postimplante, eventos cardiovasculares (definidos como síndrome coronario agudo, insuficiencia cardíaca y accidente cerebrovascular), mortalidad cardiovascular, y modificación de grado funcional y cognitivo al final del seguimiento.

\section{Análisis estadístico}

El análisis estadístico se realizó con el programa ISPSS versión 18.0 (SPSS Inc., Chicago, Illinois). El test empleado para comprobar la distribución normal de los datos fue Kolmogorov-Smirnov. Los datos se expresan como media \pm desviación típica (SD). Las variables edad en el momento del implante del marcapasos y FEVI no siguieron una distribución normal (mediana 82 años-rango intercuartílico 6 y media 60\%-rango intercuartílico 3\%, respectivamente); sin embargo utilizamos parámetros de centralización y dispersión para su análisis estadístico, debido a un coeficiente de variación menor del $33 \%$ y al importante tamaño muestral. Para comparar variables continuas entre los dos grupos empleamos el test de Student para muestras independientes (o el test $U$ de Mann-Whitney si no existía distribución normal). Para comparar más de dos medias,
Tabla 1. Análisis descriptivo de características basales

\begin{tabular}{|l|c|}
\hline $\begin{array}{l}\text { Edad al implante del marcapasos (años) } \\
\begin{array}{l}\text { Sexo } \\
- \text { Vujer }\end{array}\end{array}$ & $\mathbf{8 2 . 1} \pm \mathbf{5 . 1}$ \\
\hline Diabetes mellitus & $146(47.4 \%)$ \\
\hline Dislipemia & $106(34.6 \%)$ \\
\hline Hipertensión arterial & $115(37.3 \%)$ \\
\hline EPOC & $270(87.7 \%)$ \\
\hline Estenosis mitral moderada-severa & $40(13 \%)$ \\
\hline Insuficiencia mitral moderada-severa & $4(1.3 \%)$ \\
\hline Estenosis aórtica moderada-severa & $26(8.4 \%)$ \\
\hline Insuficiencia aórtica moderada-severa & $9(2.9 \%)$ \\
\hline Insuficiencia tricuspídea moderada-severa & $12(3.9 \%)$ \\
\hline Hipertensión pulmonar moderada-severa & $18(5.9 \%)$ \\
\hline Fibrilación auricular paroxística/persistente & $22(7.2 \%)$ \\
\hline Fibrilación auricular permanente (crónica) & $61(19.8 \%)$ \\
\hline Cardiopatía isquémica & $46(14.9 \%)$ \\
\hline Insuficiencia cardíaca crónica & $50(16.2 \%)$ \\
\hline ERC moderada-severa (EGFR $\leq 45$ ml/min) & $22(7.2 \%)$ \\
\hline
\end{tabular}

empleamos el test ANOVA, y el test de Tukey para el análisis post hoc. El test de Chi-cuadrado (o test de Fisher si un subgrupo tenía representación inferior a 5 individuos) se empleó para valorar diferencias entre variables cualitativas. Analizamos predictores de mortalidad mediante regresión logística, introduciendo mortalidad por cualquier causa al final del periodo de seguimiento como variable dependiente, y factores de riesgo cardiovascular, valvulopatías, modos de estimulación, insuficiencia cardíaca e insuficiencia renal crónica, como variables independientes. Asimismo, efectuamos un estudio de supervivencia mediante el método de Kaplan-Meier con análisis comparativo de supervivencia mediante el test de Log-Rank. Se consideró la significación estadística con un valor $p=0.05$.

\section{Resultados}

\section{Características basales}

En la población total del estudio, la edad media en el momento del implante del marcapasos fue $82.1 \pm 5.1$ años (52.6\% varones), significativamente superior en el grupo de estimulación ventricular $(84.4 \pm 5.3$ vs. $81 \pm 4.7$; 
Tabla 2. Análisis comparativo de características basales

\begin{tabular}{|c|c|c|c|}
\hline & Marcapasos ventricular & Marcapasos fisiológico & $\mathbf{p}$ \\
\hline Edad al implante del marcapasos (años) & $84.24 \pm 5.32$ & $81.07 \pm 4.71$ & $<0.001$ \\
\hline $\begin{array}{l}\text { Sexo } \\
\text { - Mujer } \\
\text { - Varón }\end{array}$ & $\begin{array}{l}42(42.4 \%) \\
57(57.6 \%)\end{array}$ & $\begin{array}{l}104(49.8 \%) \\
105(50.2 \%)\end{array}$ & 0.228 \\
\hline Diabetes mellitus & $33(33.3 \%)$ & $73(34.9 \%)$ & 0.783 \\
\hline Dislipemia & $32(32.3 \%)$ & $83(39.7 \%)$ & 0.211 \\
\hline Hipertensión arterial & $88(88.9 \%)$ & $182(87.1 \%)$ & 0.652 \\
\hline EPOC & $18(18.2 \%)$ & $22(10.6 \%)$ & 0.064 \\
\hline Estenosis mitral moderada-severa & $3(3 \%)$ & $1(0.5 \%)$ & 0.099 \\
\hline Insuficiencia mitral moderada-severa & $15(15.2 \%)$ & $11(5.3 \%)$ & 0.004 \\
\hline Estenosis aórtica moderada-severa & $4(4 \%)$ & $5(2.4 \%)$ & 0.477 \\
\hline Insuficiencia aórtica moderada-severa & $3(3 \%)$ & $9(4.3 \%)$ & 0.757 \\
\hline Insuficiencia tricuspídea moderada-severa & $13(13.1 \%)$ & $5(2.4 \%)$ & $<0.001$ \\
\hline Hipertensión pulmonar moderada-severa & $13(13.1 \%)$ & $9(4.3 \%)$ & 0.005 \\
\hline Fibrilación auricular paroxística/persistente & $20(20.2 \%)$ & $41(19.6 \%)$ & 0.904 \\
\hline Fibrilación auricular permanente (crónica) & $45(45.5 \%)$ & $1(0.5 \%)$ & $<0.001$ \\
\hline Cardiopatía isquémica & $13(13.1 \%)$ & $37(17.7 \%)$ & 0.310 \\
\hline Insuficiencia cardíaca crónica & $12(12.1 \%)$ & $10(4.8 \%)$ & 0.021 \\
\hline ERC moderada-severa (EGFR $\leq 45 \mathrm{ml} / \mathrm{min}$ ) & $28(28.3)$ & $46(22 \%)$ & 0.229 \\
\hline
\end{tabular}

$p<0.001)$. La indicación más frecuente de implante de marcapasos fue el bloqueo auriculoventricular completo (44.3\%), seguido de la fibrilación auricular lenta o bloqueada (16.7\%) y del bloqueo auriculoventricular de segundo grado sintomático (13.8\%). El $35.1 \%$ de los pacientes recibió un marcapasos monocameral y el $64.6 \%$ bicameral. El modo de estimulación más utilizado fue el DDD (38.6\%), seguido del VVI (32.1\%) y, muy de cerca, el VDD (27.6\%) (Tablas 1 y 2 ).

El subgrupo de pacientes octogenarios con primoimplante de marcapasos lo constituyeron 186 pacientes, con una edad media de $85 \pm 4$ años (51.1\% varones). La alteración electrocardiográfica más frecuente que condicionó el implante seguía siendo el bloqueo auriculoventricular avanzado, mientras que el dispositivo más empleado fue el VVI (38.7\%), seguido del VDD (38.2\%) $y$, en tercer lugar, el DDD (21.5\%). En los octogenarios con bloqueo auriculoventricular completo, el modo VDD fue el más ampliamente utilizado (56.4\%), seguido del VVI (25.5\%) y el DDD (18.1\%) (Fig. 1). A destacar también una proporción de insuficiencia tricuspídea e hipertensión pulmonar superior en dispositivos VVI (12.5 vs.
$0,9 \%$ y 15.3 vs. $3,5 \%$, respectivamente). No hubo diferencias significativas en la puntuación del Índice de Barthel ni la Escala CRF antes del implante de marcapasos (mediana $95 \pm 76,2 ; p=0.669 ; y$ mediana $2 \pm 4$; $p=0.749$, respectivamente).

\section{Seguimiento}

En la muestra total, la tasa de mortalidad global fue $21.1 \%$ (con un tiempo medio de seguimiento de 3.5 años), lo que corresponde aproximadamente al doble que la esperada en individuos de similar edad y sexo en la población general, según datos del Instituto Nacional de Estadística (mortalidad global en pacientes con primoimplante de marcapasos en Ciudad Real, 20$25 \%$ [mortalidad en individuos de similar edad y sexo en población general, 9-10\%]) (INEbase, tablas de mortalidad de la población de España por año, sexo, edad y funciones). Únicamente 18 pacientes (5.8\%) presentaron complicaciones durante el postoperatorio inmediato o temprano, de los cuales, $10(56 \%), 6(33 \%)$ y $2(11 \%)$ correspondieron a pacientes sujetos a modos 


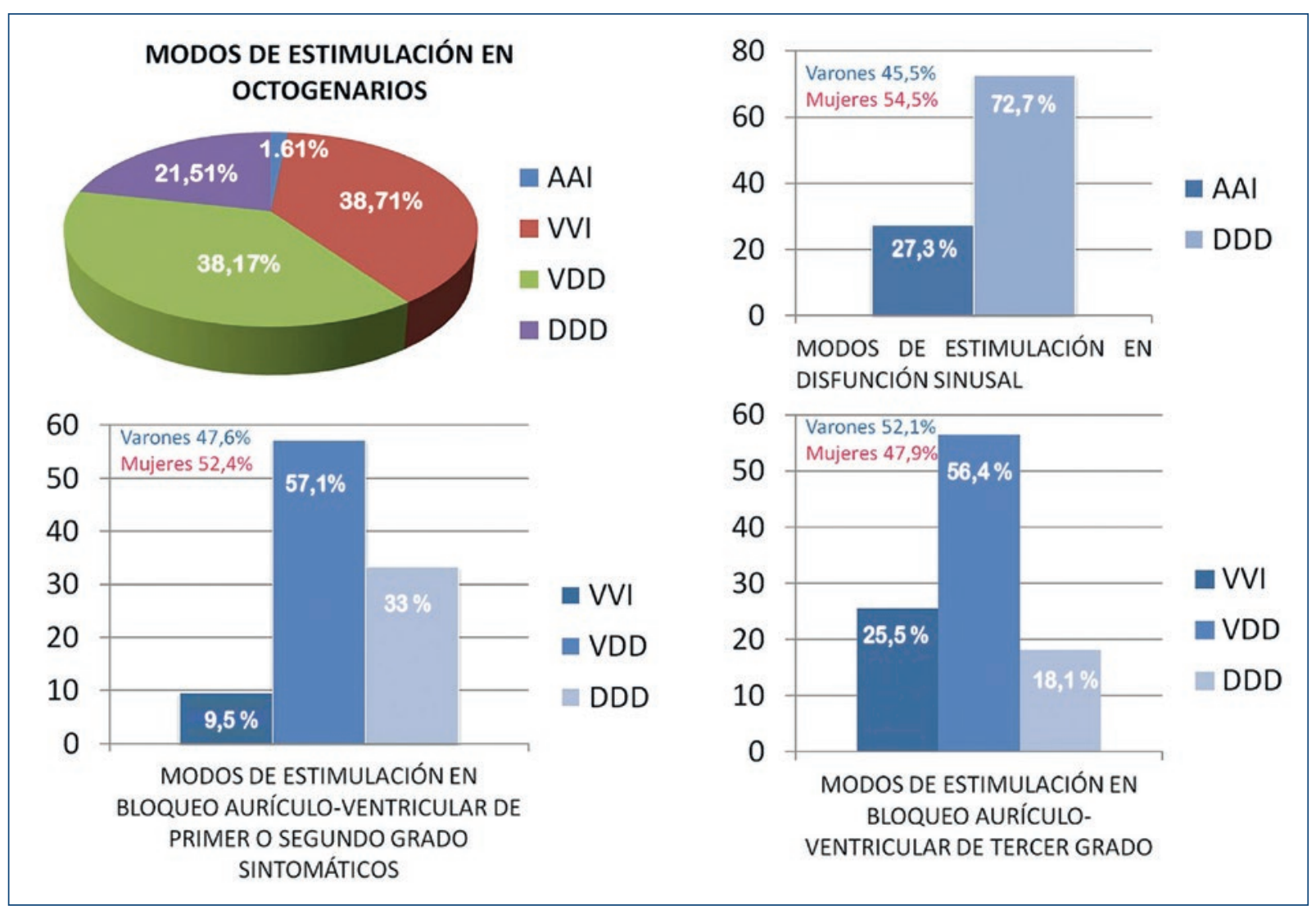

Figura 1. Modos de electroestimulación en pacientes octogenarios portadores de marcapasos.

de estimulación DDD, VDD y VVI, respectivamente. No hubo diferencias en el análisis estadístico global en complicaciones ni eventos cardiovasculares en función del modo de estimulación. Aunque detectamos mayor tasa de episodios de fibrilación auricular en dispositivos fisiológicos, la mayoría de estos episodios fueron detectados en el análisis del electrograma de marcapasos, por lo tanto, no resulta una comparación válida debido a la ausencia de detección de actividad auricular de los dispositivos VVI. La mortalidad cardiovascular y por cualquier causa al final del periodo de seguimiento fue superior en dispositivos ventriculares que en fisiológicos (33.3 vs. $15.3 \%$; OR: 0.4; IC 95\%: 0.2- 0.6; $\mathrm{p}<0.001$ ) (Tabla 3).

En los individuos octogenarios no detectamos diferencias significativas en la tasa de complicaciones postimplante, fibrilación auricular, síndrome coronario agudo ni insuficiencia cardíaca (4.2 vs. $8.6 \%$; OR: 2.2; IC 95\%: 0.2-19; $p=0.674$; 0 vs. $7.1 \%$; OR: 0.7 ; IC $95 \%$ : $0.6-0.8 ; p=0.324 ; 4.2$ vs. $1.4 \%$; OR 0.3 ; IC $95 \%$ : $0.02-$ $5.5 ; p=0.249$, y 20.8 vs. $12.9 \%$; OR: 0.6; IC 95\%: 0.2$1.9 ; p=0.415$, respectivamente). Sin embargo, la tasa de mortalidad por cualquier causa a 2 y 3 años y al final del periodo de seguimiento fue superior en los dispositivos ventriculares con respecto a los fisiológi$\cos (50$ vs. $14.3 \%$; OR: 0.2; IC 95\%: 0.06-0.5; $p=0.001$; 63.6 vs. $20 \%$; OR: 0.1 ; IC 95\%: 0.04-0.6; $p=0.007$, y 54.2 vs. $18.6 \%$; OR: 0.2 ; IC $95 \%$ : 0.07-0.5; $p=0.001$, respectivamente). Estos resultados se mantuvieron al excluir los pacientes con valvulopatías graves al inicio del análisis. En los pacientes octogenarios con bloqueo auriculoventricular avanzado, los resultados fueron similares: no detectamos diferencia significativa en la tasa de reingresos por síndrome coronario agudo $(p=0.249)$ e insuficiencia cardíaca $(p=0.415)$ en función del modo de estimulación (VVI vs. VDD vs. DDD), si bien, encontramos mayor mortalidad global a los 2 años postimplante $(p=0.001), 3$ años postimplante $(p=0.004)$ y final del periodo de seguimiento ( $p=0.001)$ en los dispositivos ventriculares con respecto a los VDD y DDD (Fig. 2).

Un análisis de subgrupos ajustado por edad, en el que comparamos individuos con bloqueo avanzado y electroestimulados con modo VVI con los que recibieron modo VDD, no objetivó diferencias significativas en la tasa de reingresos por insuficiencia cardíaca ni 
Tabla 3. Morbimortalidad cardiovascular global

\begin{tabular}{|l|c|c|c|c|}
\hline & $\begin{array}{c}\text { Marcapasos } \\
\text { ventricular }\end{array}$ & $\begin{array}{c}\text { Marcapasos } \\
\text { fisiológico }\end{array}$ & OR \\
\hline Mortalidad por cualquier causa a 1 año & $16(16.2 \%)$ & $13(6.2 \%)$ & $0.344(0.158-0.747)$ & 0.005 \\
\hline Mortalidad cardiovascular a 1 año & $5(5.1 \%)$ & $6(2.9 \%)$ & $0.556(0.165-1.867)$ & 0.336 \\
\hline Mortalidad por cualquier causa a 2 años & $28(28.3 \%)$ & $23(11 \%)$ & $0.314(0.169-0.580)$ \\
\hline Mortalidad cardiovascular a 2 años & $9(9.1 \%)$ & $6(2.9 \%)$ & $0.296(0.102-0.855)$ & 0.018 \\
\hline Mortalidad por cualquier causa a 3 años & $6(13 \%)$ & $5(3.7 \%)$ & $0.447(0.209-0.954)$ & 0.035 \\
\hline Mortalidad cardiovascular a 3 años & $6(13 \%)$ & $5(3.7 \%)$ & $0.256(0.074-0.885)$ & 0.022 \\
\hline Mortalidad por cualquier causa a fin del seguimiento & $33(33.3 \%)$ & $32(15.3 \%)$ & $0.362(0.206-0.635)$ \\
\hline Mortalidad cardiovascular a fin del seguimiento & $11(11.1 \%)$ & $8(3.8 \%)$ & $0.318(0.124-0.819)$ \\
\hline Complicaciones postimplante de marcapasos & $2(2 \%)$ & $15(7.2 \%)$ & $4.021(0.906-17.842)$ & 0.013 \\
\hline Episodios de fibrilación auricular "de novo" & $0(0 \%)$ & $17(8.1 \%)$ & $0.660(0.608-0.717)$ \\
\hline Síndrome coronario agudo & $1(1 \%)$ & $3(1.4 \%)$ & $1.413(0.145-13.756)$ \\
\hline Insuficiencia cardíaca aguda & $21(21.2 \%)$ & $25(12 \%)$ & $0.507(0.268-0.960)$ \\
\hline Accidente cerebrovascular (ACV) & $6(6.1 \%)$ & $6(2.9 \%)$ & $0.458(0.144-1.458)$ \\
\hline
\end{tabular}

eventos cardiovasculares, si bien, al igual que en el análisis global, los pacientes presentaron una mayor mortalidad global al fin del periodo de seguimiento.

El análisis multivariante definió insuficiencia cardíaca crónica y marcapasos VVI como factores predictores independientes de mortalidad de los pacientes octogenarios con indicación de marcapasos $(p=0.034$; $p=0.024$, y $p=0.001$, respectivamente). El tiempo mediano hasta la muerte por cualquier causa fue 0.9 años, sin diferencias entre los modos de estimulación ventricular $(1.01 \pm 0.5$ años [mediana \pm amplitud intercuartílica]) vs. fisiológico $(0.9 \pm 1.6$ años [mediana \pm amplitud intercuartílica]) ( $p=0.608)$.

Asimismo, realizamos un subanálisis ajustado por edad y excluyendo aquellos pacientes con valvulopatías graves, para estudiar la tasa de hospitalizaciones por insuficiencia cardíaca y mortalidad cardiovascular y global a largo plazo. Dicho subestudio objetivó diferencias en el último parámetro a favor de los pacientes con electroestimulación fisiológica versus ventricular, al igual que ocurría en el análisis general.

En cuanto a la relación de la electroestimulación con la modificación del grado funcional, analizamos 82 pacientes ancianos de los que pudimos cuantificar su Índice de Barthel y la Escala CRF en el momento del implante, y 3 años después del mismo, con una edad media de $84.6 \pm$ 2.9 años (43.9\% hombres y $56.1 \%$ mujeres). No detectamos diferencias significativas en la variación de Índice de Barthel y la Escala CRF en pacientes octogenarios con disfunción sinusal, bloqueo auriculoventricular de segundo grado sintomático, trastorno de la conducción intraventricular y fibrilación auricular. De hecho, los individuos mayores de 80 años con bloqueo auriculoventricular completo presentaron una variación media del Índice de Barthel de $-17.7 \pm 27.1(p<0.001)$ y una variación media de la Escala CRF de $0.9 \pm 1.3(p<0.001)$ (Fig. 3).

\section{Discusión}

El incremento progresivo de la edad en el momento del implante de marcapasos conlleva un aumento de la comorbilidad y la morbimortalidad, hallazgo que hemos confirmado con los resultados expuestos. La edad media de la mayoría de los estudios observacionales y experimentales relacionados con la estimulación cardíaca permanente no supera los 78 años, y en casi todos ellos el subgrupo de pacientes octogenarios se encuentra absolutamente infrarrepresentado ${ }^{3-6}$. Sin embargo, en nuestra muestra, la edad media fue superior y, probablemente, refleja mejor la situación real del implante de marcapasos en la actualidad.

La diferencia en cuanto a la tasa de utilización de los marcapasos VDD entre la población de nuestro estudio y la población general puede explicarse por la exclusión de los individuos menores de 70 años y la tendencia a intentar mantener la sincronía auriculoventricular en pacientes ancianos con comorbilidad alta y bloqueo avanzado, en nuestra unidad. 


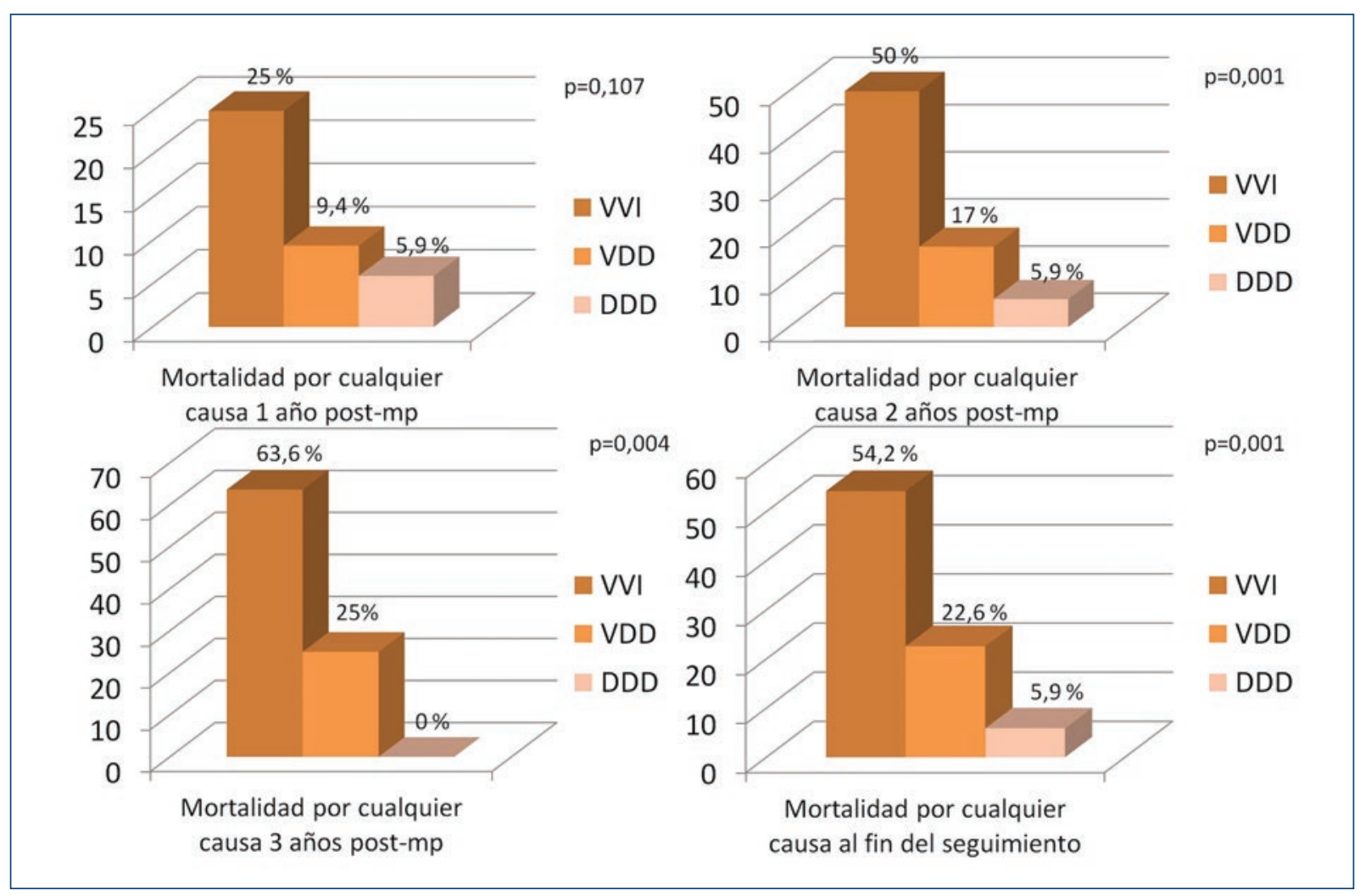

Figura 2. Mortalidad cardiovascular y por cualquier causa en pacientes octogenarios con primoimplante de marcapasos.

Existe controversia acerca de si el modo VDD ofrece ventajas sobre el VVI en este contexto en relación con el mantenimiento de la sincronía auriculoventricular. Algunos estudios sugieren un cierto beneficio en la utilización del modo de estimulación VDD en pacientes con función sinusal conservada y BAV, aunque existe un riesgo mayor de pérdida del sensado auricular con el paso del tiempo ${ }^{29}$. Por otro lado, tampoco existen diferencias claras de morbimortalidad del VDD con respecto al DDD ${ }^{11-14}$. En nuestro estudio no detectamos diferencias en la tasa de complicaciones postimplante ni eventos cardiovasculares (síndrome coronario agudo y/o hospitalización por insuficiencia cardíaca) en individuos octogenarios con bloqueo avanzado en función del modo de estimulación (VVI, VDD, DDD) $(n=94)$, si bien, objetivamos una mayor tasa de mortalidad a medio-largo plazo en dispositivos ventriculares con respecto a fisiológicos. El análisis multivariante que efectuamos definió el modo de estimulación VVI como predictor independiente de mortalidad en este subgrupo de pacientes. La limitación que condiciona tener un tamaño muestral relativamente bajo de pacientes octogenarios con bloqueo completo (94) nos obliga a ser prudentes para la elaboración de conclusiones firmes, si bien, nos permite formular la hipótesis de que el marcapasos VDD puede ser una alternativa válida y útil en pacientes ancianos con indicación de marcapasos por bloqueo avanzado y elevada comorbilidad.

En nuestro análisis no detectamos mejoría significativa del grado funcional o cognitivo de los enfermos a medio-largo plazo, tal que incluso en algunos pacientes, como los pacientes con bloqueo avanzado, el grado funcional era significativamente peor posteriormente al implante de marcapasos. Planteamos que en pacientes ancianos y muy ancianos debemos orientar nuestra actuación en el campo de las bradiarritmias en función de lo que las guías de electroestimulación así refieren. Sin embargo, es probable que debamos tener en cuenta otras variables como la probabilidad de modificación del grado de dependencia tanto física como psicológica. Probablemente, una media de 3.5 años de seguimiento sea demasiado larga para el análisis de este grado funcional en una muestra con una edad media tan alta. Esto podría explicar en parte los resultados tan poco alentadores en este contexto y sería la base para futuros estudios prospectivos, con tiempos de seguimiento más cortos (1 mes, 6 meses, 1 año, etc.). A la vista de estos hallazgos, debemos ser prudentes 


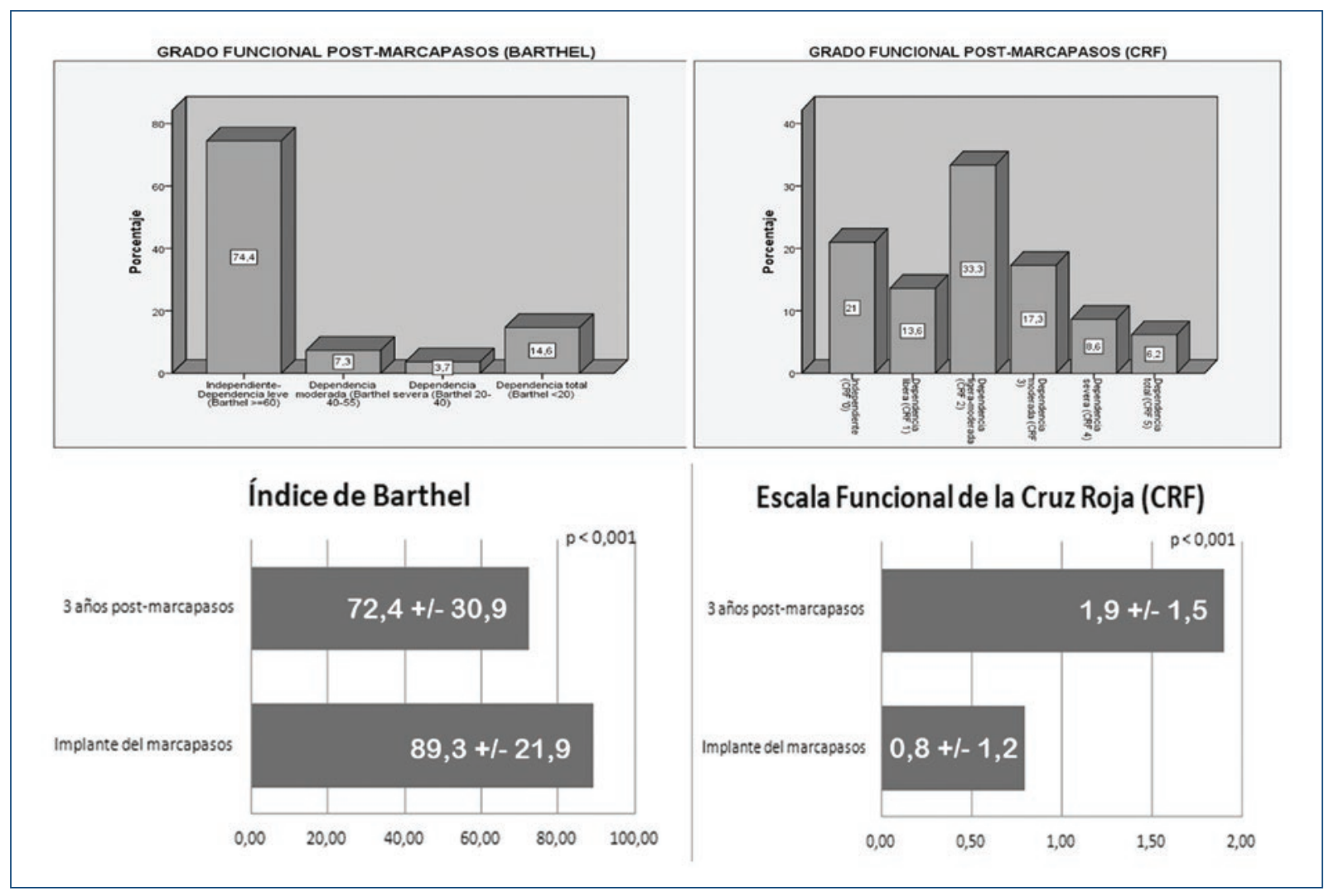

Figura 3. Variación del grado funcional en pacientes octogenarios con primoimplante de marcapasos.

en pacientes octogenarios y no dar falsas expectativas, ya que no parece previsible encontrar una mejoría significativa de la capacidad funcional y cognitiva al menos a medio plazo. Debemos asumir que el deterioro funcional va a depender de otros factores, y no solo de la alteración intrínseca a nivel del sistema de excitación-conducción cardíaco.

\section{Limitaciones}

La indicación de implante de dispositivo ventricular (más allá de la fibrilación auricular lenta o bloqueada) la realizó el operador en cuestión basándose en la comorbilidad del paciente y riesgo de complicaciones, pero en ausencia de parámetros objetivos que cuantificasen ambos, lo que puede inducir a un sesgo de selección. Asimismo, la edad media en el momento del implante de marcapasos fue significativamente mayor en los dispositivos ventriculares que en los fisiológicos, lo cual puede artefactar de manera relevante los resultados en cuanto a morbimortalidad, sobre todo teniendo en cuenta que partimos de una población con comorbilidades importantes. Por último, el tamaño muestral relativo que obtuvimos al analizar los pacientes octogenarios con bloqueo $(n=94)$ y aquellos a quienes les pudimos realizar valoración geriátrica previa y postimplante de marcapasos $(n=82)$ (de la muestra inicial de 308 y 186 octogenarios), no nos permite establecer conclusiones firmes sino formular nuevas hipótesis que deberán ser corroboradas en futuros estudios.

\section{Conclusiones}

La morbimortalidad cardiovascular en pacientes mayores de 80 años portadores de marcapasos es elevada y muy superior a la de la población general de igual edad. Con los resultados de nuestro estudio podría plantearse la utilización de dispositivos bicamerales, como el VDD, para garantizar cierto grado de sincronía auriculoventricular en pacientes octogenarios con bloqueo auriculoventricular persistente y función sinusal conservada, sin aumentar con ello la morbimortalidad cardiovascular.

La mejoría del grado funcional físico o cognitivo a largo plazo es inexistente, tal y como hemos demostrado. Tanto es así, que incluso existen subgrupos de pacientes en 
los que este parámetro es significativamente peor 3 años después del implante. Desde nuestro grupo de trabajo creemos que es necesario realizar estudios prospectivos en los que se analicen la variación del grado funcional a corto plazo en pacientes ancianos y muy ancianos (comparando por ejemplo el Índice de Barthel inmediatamente antes del implante, con el registrado un mes, tres meses o seis meses postimplante), debido a que la elevada vida media de nuestros pacientes (con sus consiguientes comorbilidades y limitaciones) puede artefactar los resultados a largo plazo.

\section{Conflicto de intereses}

Ninguno.

\section{Financiamiento}

Ninguna.

\section{Responsabilidades éticas}

Protección de personas y animales. Los autores declaran que para esta investigación no se han realizado experimentos en seres humanos ni en animales.

Confidencialidad de los datos. Los autores declaran que han seguido los protocolos de su centro de trabajo sobre la publicación de datos de pacientes.

Derecho a la privacidad y consentimiento informado. Los autores han obtenido el consentimiento informado de los pacientes y/o sujetos referidos en el artículo. Este documento obra en poder del autor de correspondencia.

\section{Bibliografía}

1. Brignole M, Auricchio A, Baron-Esquivias G, Bordachar P, Boriani G, Breithardt OA, et al.; ESC Committee for Practice. Guidelines (CPG). 2013 ESC Guidelines on cardiac pacing and cardiac resynchronization therapy: the Task Force on cardiac pacing and resynchronization therapy of the European Society of Cardiology (ESC). Developed in collaboration with the European Heart Rhythm Association (EHRA). Eur Heart J. 2013;34(29):2281-329.

2. Cano O, Pombo M, Fidalgo ML. XIV Informe Oficial de la Sección de Estimulación Cardiaca de la Sociedad Española de Cardiología (2016). Rev Esp Cardiol. 2017;70(12):1083-97.

3. Andersen HR, Thuesen L, Bagger JP, Vesterlund T, Thomsen PE. Prospective randomised trial of atrial versus ventricular pacing in sick-sinus syndrome. Lancet. 1994;344(8936):1523-8.

4. Lamas GA, Pashos CL, Normand SL, McNeil B. Permanent pacemaker selection and subsequent survival in elderly Medicare pacemaker recipients. Circulation. 1995;91(4):1063-9.

5. Lamas GA, Lee KL, Sweeney MO, Silverman R, Leon A, Yee R, et al. Ventricular pacing or dual-camber pacing for sinus-node dysfuncion. N Engl J Med. 2002;346(24):1854-62.
6. Nielsen JC, Thomsen PE, Hojberg S, Moller M, Vesterlund T, Dalsgaard D, et al. A comparison of single-lead atrial pacing with dual- chamber pacing in sick sinus syndrome. Eur Heart J. 2011;32(6):686-96.

7. Breivik K, Ohmn OJ, Segadal L. Sick sinus syndrome treated with permanent pacemaker in 109 patients. A follow-up study. Acta Med Scand. 1979;206:153-9.

8. Lamas GA, Lee KL, Sweeney MO, Silverman R, Leon Y, Yee R, et al. Ventricular pacing or dual-camber pacing for sinus-node dysfuncion. N Engl J Med. 2002;346(24):1854-62

9. Connolly SJ, Kerr CR, Gent M, Roberts RS, Yusuf S, Gillis AM, et al Effects of physiologic pacing versus ventricular pacing on the risk of stroke and death due to cardiovascular causes. Canadian Trial of Physiologic Pacing Investigators. N Engl J Med. 2000;342(19):1385-91.

10. Toff WD, Camm AJ, Skehan JD; United Kingdom Pacing and Cardiovascular Events Trial Investigators (UKPACE). Single-chamber versus dual-chamber pacing for high-grade atrioventricular block. N Engl J Med. 2005;353(2):145-55.

11. Huang M, Krah AD, Yee R, Klein GJ, Skanes AC. Optimal pacing for symptomatic AV block: a comparison of VDD and DDD pacing. Pacing Clin Electrophysiol. 2004;27(1):19-23.

12. Huang CC, Tuan TC, Fong MC, Lee WS, Kong CW. Predictors of inappropriate atrial sensing in long- term VDD-pacing systems. Europace. 2010;12(9):1251-5

13. Nowak $B$, Przibille O. Atrial undersensing in VDD pacing: should we blame calcium-cannel blockers? Europace. 2010;12(9):1205-6.

14. Marchandise S, Scavée C, le Polain de Warux JB, de Meester C, Vanoverschelde JL, Debbas N. Long-term follow-up of DDD and VDD pacing: a prospective non-randomized single-centre comparison of patients with symptomatic atrioventricular block. Europace. 2012;14(4):496-501.

15. Wiegand UK, Bode F, Schneider R, Brandes A, Haase $H$, Katus HA, et al. Development of sinus node disease in patients with AV block: implications for a single lead VDD pacing. Heart. 1999;81(6):580-5.

16. Kühne M, Schaer B, Kaufmann C, Moulay N, Cron T, Cueni T, et al. A randomized trial comparing two different approaches of pacemaker selection. Europace. 2007;9(12):1185-90.

17. Wiegand UK. Single-lead VDD pacing- a serious alternative for atrioventricular synchronous pacing with atrioventricular block? Europace. 2012;14(4):461-2.

18. Garillo R, Moreno M. Marcapasos cardíacos. Estimulación desde el ventrículo derecho. Beneficios y perfjuicios a la luz de la experiencia actual. Rev Costarr Cardiol. 2011;13(1):19-22.

19. Wilkoff BL, Cook JR, Epstein AE, Greene HL, Hallstrom AP, Hsia H, et al. Dual-chamber pacing or ventricular backup pacing in patients with an implantable defibrillator: the Dual Chamber and VVI Implantable Defibrillator (DAVID) Trial. JAMA. 2002;288:3115-23.

20. Andersen HR, Nielsen JC, Thomsen PE, Thuesen L, Mortensen PT, Vesterlund $\mathrm{T}$, et al. Long-term follow-up of patients from a randomised trial of atrial versus ventricular pacing for aick-sinus syndrome. Lancet. 1997;350(9086):1210-6.

21. De Juan J, Olagüe J, Morell S, García I. Estimulación cardiaca con marcapasos. Seguimiento del paciente con marcapasos. Disfunciones del sistema de estimulación. Efectos de indicación o programación incorrecta: síndrome del marcapasos. Rev Esp Cardiol Supl. 2007:7:126G-44G.

22. Rosenqvist $M$, Brandt $J$, Shüller $H$. Long-term pacing in sinus node disease: effects of stimulation mode on cardiovascular morbidity and mortality. Am Heart J. 1988;116:16-22.

23. Lamas GA, Orav EJ, Stambler BS, Ellenbogen KA, Sgarbossa EB, Huang SK, et al. Quality of life and clinical outcomes in elderly patients treated with ventricular pacing as compared with dual-chamber pacing. Pacemaker Selection in the Elderly Investigators (PASE). $\mathrm{N}$ Engl J Med. 1988;338(16):1097-104.

24. Pareja T, Castro J, Jiménez MP, Balaguer R. Paciente anciano portador de marcapasos: características y evolución en función del tipo de dispositivo empleado. Rev Esp Geriatr Gerontol. 2007;42(3):149-57.

25. Cabrerizo $P$, Pérez N, Sánchez M, Herreros M, Cerrón B, Núñez J, et al. 6010-15- Supervivencia de los pacientes ancianos sometidos a un implante de marcapasos. Rev Esp Cardiol. 2011;64Supl 3:268.

26. Durán $\mathrm{O}$, Amor A, Jiménez-Candil J, Rodríguez T, Hernández JM, Moríñigo JL, et al. 7004-18- Complicaciones asociadas al implante de marcapasos en ancianos: la importancia de la edad. Resultados de una serie contemporánea. Rev Esp Cardiol. 2014;67 Supl 1:1087.

27. Collin C, Wade DT, Davies S, Horne V. The Barthel LADL Index: a reliability study. Int Disabil Stud. 1988;10(2):61-3.

28. Trigás-Ferrín M, Ferreira-González L, Meijide-Míguez $H$. Escalas de valoración funcional en el anciano. Galicia Clin. 2011;72(1):11-6.

29. Wiegand UK, Potratz J, Bode F, Schneider R, Peters W, Bonnemeier H, et al. Age dependency of sensing performance and AV synchrony in single lead VDD pacing. Pacing Clin Electrophysiol. 2000;23(5):863-9. 